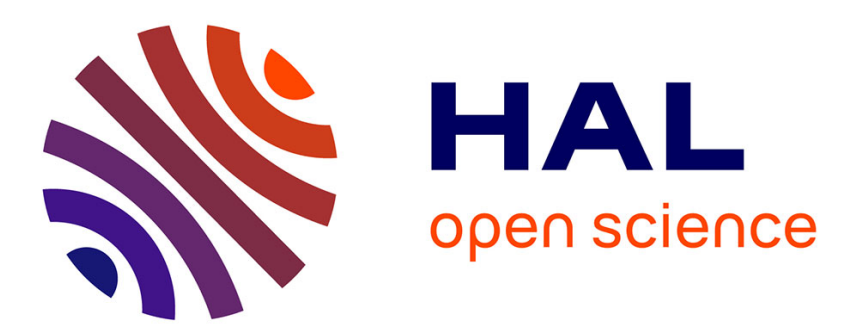

\title{
Contribution des plasmons de surface au rayonnement lumineux émis par des jonctions tunnel MIM réalisées sur réseaux holographiques
}

S.M. Moulessehoul, A. Septier

\section{- To cite this version:}

S.M. Moulessehoul, A. Septier. Contribution des plasmons de surface au rayonnement lumineux émis par des jonctions tunnel MIM réalisées sur réseaux holographiques. Revue de Physique Appliquée, 1984, 19 (7), pp.503-512. 10.1051/rphysap:01984001907050300 . jpa-00245221

HAL Id: jpa-00245221

https://hal.science/jpa-00245221

Submitted on 1 Jan 1984

HAL is a multi-disciplinary open access archive for the deposit and dissemination of scientific research documents, whether they are published or not. The documents may come from teaching and research institutions in France or abroad, or from public or private research centers.
L'archive ouverte pluridisciplinaire HAL, est destinée au dépôt et à la diffusion de documents scientifiques de niveau recherche, publiés ou non, émanant des établissements d'enseignement et de recherche français ou étrangers, des laboratoires publics ou privés. 


\title{
Contribution des plasmons de surface au rayonnement lumineux émis par des jonctions tunnel MIM réalisées sur réseaux holographiques
}

\author{
S. M. Moulessehoul et A. Septier \\ Laboratoire de Physique du Vide (*), CNAM, 292, rue St-Martin, 75141 Paris Cedex 03, France
}

(Reçu le 30 janvier 1984, révisé le 30 mars, accepté le 3 avril 1984)

\begin{abstract}
Résumé. - L'analyse spectrale dans différentes directions angulaires de la lumière émise par des jonctions tunnel de type $\mathrm{Al}-\mathrm{Al}_{2} \mathrm{O}_{3}-\mathrm{Ag}$ réalisées sur des réseaux holographiques et refroidies vers $T=100 \mathrm{~K}$ permet de déterminer les courbes de dispersion des différents ordres émis par les ondes de plasma de surface rendues radiatives par la modulation périodique de la surface. Les contributions respectives des plasmons excités sur la surface externe d'Ag et à l'interface $\mathrm{Al}_{2} \mathrm{O}_{3}-\mathrm{Ag}$ ont pu être déterminées par une mesure absolue de l'intensité émise.
\end{abstract}

\begin{abstract}
A study of optical spectra and angular dependence of light emitted from $\mathrm{Al}^{-} \mathrm{Al}_{2} \mathrm{O}_{3}-\mathrm{Ag}_{\mathrm{g}}$ tunnel diodes fabricated on diffraction gratings allowed a determination of the dispersion curves of the surface plasma waves (SPW) excited by electrons in the structure. Respective contribution of SPW propagating on the external surface, and at the $\mathrm{Al}_{2} \mathrm{O}_{3}-\mathrm{Ag}$ interface have been determined by absolute measurements of the radiated light intensity.
\end{abstract}

\section{Introduction.}

On appelle diode MIM (Métal-Isolant-Métal) une structure formée d'une couche mince d'isolant placée entre deux électrodes métalliques. Ces structures sont souvent utilisées pour étudier les phénomènes de conduction électrique dans les matériaux isolants. Lors d'études menées sur des diodes où l'isolant a des épaisseurs allant de quelques dizaines à quelques centaines de nanomètres, on s'est aperçu qu'il existait une faible luminescence lorsqu'on appliquait au dispositif une polarisation de quelques volts $[1,2]$. Cette luminescence n'est visible qu'avec les forts courants obtenus après "formation " des diodes fonctionnant en régime de résistance négative. Malgré les études récentes qui lui ont été consacrées [3-5], son origine est encore controversée.

Il est également possible d'obtenir de forts courants dans des structures MIM en utilisant l'effet tunnel à travers des isolants très minces $(2$ à $3 \mathrm{~nm})$. En contrepartie, et même avec des isolants de très bonne qualité, la tension de polarisation ne peut guère dépasser $3 \mathrm{~V}$, avec une épaisseur d'isolant de $3 \mathrm{~nm}$. Dans ce régime de conduction tunnel, une émission lumineuse ayant des caractéristiques très différentes de la précédente

(*) ERA 660 . a été mise en évidence en 1976 [6]. Il s'agit d'une émission s'étendant dans le domaine optique, sans structure particulière, avec une énergie supérieure des photons $E=\hbar \omega_{\mathrm{m}}$ limitée par la tension de polarisation $V_{0}$ selon une relation du type : $\hbar \omega_{\mathrm{m}}=e V_{0}$ et une intensité d'autant plus forte que l'électrode externe est plus rugueuse [7].

Les auteurs supposent que l'émission est due à des ondes de plasma de surface (OPS) excitées dans la structure par les électrons circulant dans la diode, et rendues radiatives par les rugosités de surface ou d'interface. On sait en effet [8] qu'il existe, dans une structure multicouches, autant d'OPS que d'interfaces métal-isolant. Dans une diode MIM, où les électrons issus de l'une des électrodes traversent l'isolant puis l'interface isolant-métal de l'autre électrode avant de s'écouler dans cette électrode, on peut penser que l'OPS la plus intense est excitée à cette interface avec ses champs électromagnétiques concentrés dans l'isolant. Plusieurs études théoriques ont été consacrées au rayonnement de ce mode [9-11] appelé " mode lent " car l'isolant lui confère une vitesse de phase nettement plus faible que celle de l'OPS pouvant se propager à l'interface métal-vide.

Il faut souligner que, dans une diode réelle, les électrons qui subissent des chocs inélastiques à la traversée de l'isolant, ont à l'arrivée dans la $2^{\mathrm{e}}$ élec- 
trode un spectre d'énergie plus large, et qu'ils peuvent exciter des plasmons d'énergie $\hbar \omega$ telle que $0<\hbar \omega<e V_{0}$, de vecteur d'onde parallèle à la surface (mais orienté de façon quelconque) et de valeur fixée par la courbe de dispersion $(\hbar \omega, k)$ de l'OPS relative à l'interface considérée.

Pour le mode interne, on aurait une probabilité d'excitation très forte, mais les OPS ainsi créées auraient de très grands vecteurs d'onde $\mathbf{k}$ et pour les rendre radiatives, il faudrait des rugosités ayant une très faible pseudo-période $\langle\sigma\rangle(\langle\sigma\rangle\langle 10 \mathrm{~nm})$ $[9,10]$. L'intensité rayonnée serait proportionnelle au carré de l'amplitude moyenne $\langle\delta\rangle$ des rugosités, pour les faibles valeurs de $\langle\delta\rangle$, puis tendrait vers une valeur limite constante, lorsque $\langle\delta\rangle$ croît fortement [10]. Cette intensité rayonnée par la surface extérieure serait très faible dans le domaine visible et le proche infrarouge. La lumière émise ne serait pas polarisée.

L'hypothèse de la radiation, à partir du mode interne, fut encore retenue pour expliquer les observations faites sur des diodes MIM de type $\mathrm{Mg}-\mathrm{MgO}-$ $\mathrm{Ag}$, le magnésium donnant naturellement, par évaporation, des films minces très rugueux [12].

A partir de 1978 un nouveau type de rayonnement fut étudié : celui de jonctions dont l'électrode supérieure est formée de particules métalliques isolées déposées sur l'isolant (en réalité, un film métallique mince est évaporé sur les particules afin de rendre l'électrode conductrice). Signalé dans une étude expérimentale [13], ce rayonnement fut ensuite étudié théoriquement $[14,15]$, puis fit de nouveau l'objet d'études expérimentales détaillées [16, 17]. Dans ce système, les particules métalliques de 10 à $20 \mathrm{~nm}$ de diamètre déposées sur l'oxyde joueraient le rôle d'antennes, excitées par les fluctuations rapides du courant des électrons qui traversent l'oxyde par effet tunnel en y subissant de nombreux chocs inélastiques. Des résonances apparaissent dans le spectre de la radiation émise par les particules, correspondant aux modes d'OPS localisées excitées dans les particules d'or [15]. La lumière émise est partiellement polarisée. Les répartitions angulaires du rayonnement et les spectres observés sont en bon accord avec les prédictions de la théorie [16, 17].

Dans les structures MIM classiques à électrode externe continue, l'émission lumineuse observée audelà de la fréquence maximale de l'OPS interne $\left(\hbar \omega \simeq 2,2 \mathrm{eV}\right.$ pour des diodes $\mathrm{Al}-\mathrm{Al}_{2} \mathrm{O}_{3}$ - $\mathrm{Au}$ rugueuses) ne peut être expliquée qu'en faisant appel à une autre origine du rayonnement : l'excitation d'une OPS sur la face externe du dispositif, à l'interface métal-vide, rendue radiative par les rugosités.

Cette possibilité, signalée dans [18] et affirmée dans [19] fut prouvée de façon irréfutable par Kirtley et al. [20] puis Kroó et al. [21] à l'aide de diodes réalisées sur des réseaux holographiques, ayant donc une rugosité bien contrôlée de périodicité connue. L'étude expérimentale [20] initia une étude théorique appro- fondie [22] sur l'excitation de l'OPS externe, encore appelée " mode rapide " de la structure. Cette étude qui fait encore appel aux fluctuations du courant tunnel dues aux chocs inélastiques des électrons traversant la barrière située dans l'isolant, a permis de déterminer les caractéristiques essentielles du rayonnement (spectre, distribution angulaire, intensité). Une étude expérimentale détaillée, menée par Kirtley et al. [23], a confirmé en partie ces prévisions théoriques. Mais des désaccords profonds concernant l'intensité rayonnée, la loi de variation de cette intensité avec l'épaisseur de l'électrode externe, et son renforcement par abaissement de température, ont conduit ces mêmes auteurs à remettre en question le mécanisme d'excitation des OPS, et montrent que l'excitation des OPS provient essentiellement d'un couplage direct entre électrons chauds et ondes de surface, localisé dans l'électrode externe [24]. Cette hypothèse, plus réaliste que la précédente, avait déjà été émise en 1980 par Kroó et al. [19], et est confirmée par d'autres publications qui observent — dans des diodes MIM à rugosité aléatoire - que l'intensité rayonnée dépend étroitement des pertes d'énergie subies par les électrons dans le métal de l'électrode externe [25, 26].

Signalons enfin que des anomalies peuvent apparaitre dans les courbes de dispersion des ondes rayonnées observées expérimentalement, pour des réseaux aux sillons relativement profonds [27, 28].

Les nombreuses publications récentes consacrées à l'électroluminescence de diodes MIM à isolant très mince illustrent l'importance des efforts faits dans de nombreuses équipes pour bien comprendre les origines du rayonnement et tenter de le renforcer au maximum en vue d'une application pratique : la réalisation de diodes électroluminescentes utilisant les OPS comme source de lumière. De telles diodes seraient d'une réalisation plus facile que celle des diodes à semiconducteur, et se prêteraient bien à une intégration poussée. En outre la couleur pourrait varier en appliquant des tensions de polarisation différentes.

Notre travail a eu pour but de réaliser des diodes MIM à isolant très mince, pouvant supporter des tensions de polarisation supérieures à $3 \mathrm{~V}$, ayant des durées de vie importantes et des caractéristiques reproductibles.

Après mise au point d'un appareillage permettant la détection et la mesure de flux lumineux très faibles, nous avons étudié les caractéristiques du rayonnement émis par de telles diodes MIM construites sur des réseaux holographiques $[29,30]$.

\section{Les diodes MIM.}

2.1 LES RÉSEAUX SUPPORTS. - Des réseaux holographiques de pas différents $(a \approx 540,600,710 \mathrm{~nm})$ ont été réalisés sur des couches minces de résine photosensible déposée sur des plaquettes de verre de $75 \times 25 \mathrm{~mm}^{2}$, les traits des réseaux étant parallèles 
au grand côté des plaquettes. De tels réseaux présentent au moins en théorie l'avantage de fournir des surfaces de rugosité parfaitement contrôlée, ayant un profil $\delta(x)$ tel que :

$$
\delta(x)=\delta_{0} \cos \frac{2 \pi x}{a} .
$$

Dans nos réseaux, l'amplitude de modulation $\delta_{0}$ est comprise entre 10 et $25 \mathrm{~nm}$.

Nous avons déterminé $\delta_{0}$ à partir de l'intensité $I_{-1}$ diffractée dans l'ordre -1 lorsqu'on envoie sur la surface le faisceau d'un laser He-Ne polarisé, d'intensité $I_{0}$, sous un angle d'incidence $\theta_{0}$. On a en effet [31] en polarisation s pour des valeurs de $\delta_{0}$ faibles devant la longueur d'onde $\lambda$ :

$$
\delta_{0}^{2}=\frac{I_{-1}}{I_{0}}\left(\frac{\lambda}{2 \pi}\right)^{2}\left(\cos \theta_{-1} \cos \theta_{0}\right)^{-1}
$$

$\theta_{-1}$ est l'angle de diffraction correspondant à l'ordre -1 .

Nous avons obtenu ainsi :

$\delta_{0} \simeq 20 \mathrm{~nm}$ pour une série de réseaux de pas $a=540 \mathrm{~nm}$,

$\delta_{0} \simeq 13,5 \mathrm{~nm}$ pour une autre série de pas $a=710 \mathrm{~nm}$. Le par

En pratique, les taches de diffraction obtenues avec ces réseaux sont très élargies par une importante diffusion sur des rugosités aléatoires superposées au relief périodique, et qui sont visibles par examen de la surface au microscope électronique à balayage.

La surface des réseaux est recouverte par évaporation sous vide d'une couche mince d'aluminium d'épaisseur 100 à $200 \mathrm{~nm}$, sous forme d'un ruban de largeur $7 \mathrm{~mm}$, parallèle au grand côté de la plaquette.

2.2 FABRICATION DES DIODES. - La couche d'aluminium évaporée sur les réseaux constitue la première électrode. L'isolant est obtenu par oxydation de cette couche. Deux méthodes d'oxydation furent successivement employées :

- oxydation anodique dans un bain de citrate d'ammonium;

- oxydation thermique en atmosphère d'oxygène.

La première méthode permet d'obtenir une couche mince d'oxyde dense de très bonne qualité et d'épaisseur qui varie linéairement avec la tension appliquée. Mais il a été impossible d'éviter la déformation de la résine des réseaux supports durant l'électrolyse, et ce procédé a dû être abandonné.

L'oxydation thermique a été effectuée dans un four parcouru par un flux d'oxygène pendant une heure environ à une température limitée à $100^{\circ} \mathrm{C}$ pour éviter la déformation de la couche de résine. Ici, encore, l'oxyde est de très bonne qualité mais l'épais- seur finale qu'on peut obtenir est limitée à quelques $\mathrm{nm}$.

L'électrode supérieure est un film mince d'argent, d'épaisseur 30 à $35 \mathrm{~nm}$, évaporé sous vide sous forme de rubans de $5 \mathrm{~mm}$ de largeur, perpendiculaires au ruban d'aluminium. Sur un même réseau support on forme ainsi 6 diodes identiques, de surface utile $7 \times 5 \mathrm{~mm}^{2}$. Les extrémités des rubans d'argent sont renforcées par évaporation de couches d'aluminium épaisses de $150 \mathrm{~nm}$ permettant l'établissement des contacts électriques. Pour des diodes utilisées à la température ambiante, ces contacts sont obtenus par collage des fils à l'aide de colle à l'argent. Mais lorsque les diodes doivent subir des cycles de refroidissement à la température de l'azote liquide, il y a décollement des connexions ainsi réalisées. Les contacts ont alors été obtenus par pression de lamelles métalliques élastiques.

2.3 CONTRÔLE DES DIODES. - Après réalisation des diodes, l'épaisseur $e$ de l'isolant peut être obtenue de façon approchée par une mesure de capacité, la constante diélectrique de $\mathrm{Al}_{2} \mathrm{O}_{3}$ étant prise égale à $\varepsilon_{0 \mathrm{x}}=8$. Pour toutes nos diodes, l'épaisseur est $e \simeq 3 \mathrm{~nm}$.

Les diodes sont ensuite mises sous tension pour en contrôler les caractéristiques $I(V)$. Sur une même plaquette, ces caractéristiques sont pratiquement identiques. Le courant $I$ varie d'abord linéairement avec la tension appliquée $V$, pour les faibles valeurs de $V$, puis croît ensuite de façon quasi exponentielle, ce

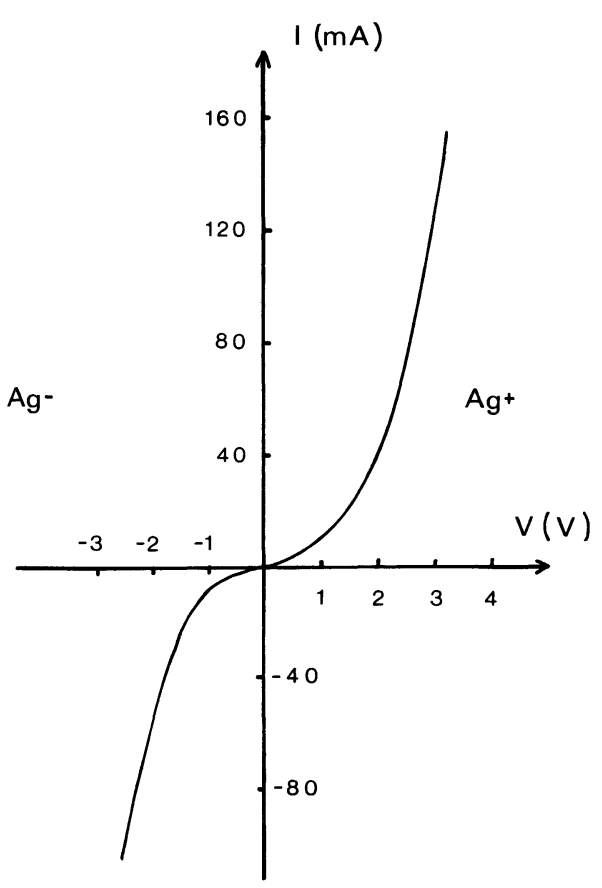

Fig. 1. - Caractéristique $I(V)$ d'une diode tunnel MIM de section $35 \mathrm{~mm}^{2}$ refroidie à $T \approx 80 \mathrm{~K}$.

[I(V) characteristics of a $35 \mathrm{~mm}^{2}$ tunnel MIM diode cooled at $T \approx 80 \mathrm{~K}$.] 
qui est typique d'un effet tunnel progressivement renforcé par le champ électrique appliqué, qui permet le passage des électrons de l'électrode métallique dans la bande de conduction de $\mathrm{Al}_{2} \mathrm{O}_{3}$.

La résistance des diodes, déterminée pour $V \simeq 0$, est d'environ $10^{3} \mathrm{ohms}$. Elle varie peu pour les 6 diodes d'une même plaquette, mais beaucoup plus pour des diodes réalisées sur des plaquettes différentes. Une faible variation de l'épaisseur d'oxyde suffit pour expliquer ces écarts.

La tension maximum $V_{0}$ applicable aux diodes sans claquage ne peut dépasser $3 \mathrm{~V}$ à la température ambiante. Par contre, un refroidissement à $T \simeq 100 \mathrm{~K}$ sous vide permet d'obtenir un régime de conduction stable et reproductible jusqu'à $3,25 \mathrm{eV}$. Le courant maximum peut alors atteindre $150 \mathrm{~mA}$, correspondant à une densité de courant $j \simeq 0,4 \mathrm{~A} / \mathrm{cm}^{2}$. Le champ électrique interne, pour une épaisseur d'isolant $e \simeq 3 \mathrm{~nm}$, est alors supérieur à $10^{7} \mathrm{~V} / \mathrm{cm}$.

La figure 1 donne une caractéristique $I(V)$ typique obtenue lorsque la diode est refroidie à une température voisine de $80 \mathrm{~K}$.

\section{Dispositif expérimental.}

La plaquette de verre supportant une série de 6 diodes est appliquée sur la surface latérale d'un cryostat à azote liquide disposé verticalement dans une enceinte à vide munie de fenêtres d'observation. La température des diodes, mesurée par thermocouple cuivreconstantan, reste toujours comprise entre 77 et $100 \mathrm{~K}$. $\mathrm{Le}$ cryostat peut pivoter autour d'un axe vertical $\mathrm{O} z$ tangent à la surface des diodes et parallèle aux traits des réseaux. Il peut également subir des translations verticales permettant d'amener successivement les diodes de la plaquette sur l'axe du système optique.

Une fente d'analyse verticale de $10 \times 2 \mathrm{~mm}^{2}$, fixée sur une des fenêtres de silice fondue située à $85 \mathrm{~mm}$ des diodes, définit l'ouverture angulaire $\Delta \theta$ du faisceau lumineux $(\Delta \theta=1,35$ degré $)$ émis par cette diode et pénétrant dans le système d'analyse. Celui-ci se compose :

- d'un miroir concave de renvoi à $90^{\circ}$, de grandissement unité,

- d'un hacheur de faisceau, fonctionnant à $600 \mathrm{~Hz}$,

- d'un polariseur,

- d'un monochromateur à réseau Jobin Yvon H-10 muni d'une fente d'entrée de $8 \times 1 \mathrm{~mm}^{2}$,

- d'un photomultiplicateur RTC couvrant le domaine spectral $300-760 \mathrm{~nm}$, associé à un dispositif de détection synchrone Keithley suivi d'une table traçante.

La largeur spectrale du faisceau analysé est de $\Delta \lambda=8 \mathrm{~nm}$.

Le système optique est tel que le flux provenant d'une surface émissive $\mathrm{d} S$ de $8 \times 1 \mathrm{~mm}^{2}$ et traversant la fente d'analyse peut atteindre le récepteur final.

L'étalonnage de l'ensemble a été effectué en remplaçant la diode MIM située sur l'axe optique par le ruban de tungstène d'une lampe étalon, dont la courbe de luminance $L(\lambda)$ a été préalablement déterminée à l'Institut National de Métrologie.

On sait que :

$$
L(\lambda)=\frac{\mathrm{d}^{3} W}{\mathrm{~d} S_{1} \mathrm{~d} \lambda \mathrm{d} \Omega}\left(\mathrm{Wm}^{-3} \mathrm{sr}^{-1}\right)
$$

où $\mathrm{d} S_{1}$ est l'aire émissive, $\mathrm{d} \Omega$ l'angle solide défini par la fente d'analyse et $\mathrm{d} \lambda$ la bande spectrale analysée. A partir du signal de sortie $V(\lambda)$, on peut déterminer la sensibilité $s(\lambda)=V(\lambda) / L(\lambda)$ du système optique pour les deux polarisations $s$ et $p$. Les courbes correspondantes sont données sur la figure 2 . En polarisation $\mathrm{p}$, le pic d'absorption à $\lambda \simeq 570 \mathrm{~nm}$ est dû à l'excitation d'une OPS dans le réseau du monochromateur.

Pour connaître le flux rayonné par une diode MIM dans le même angle solide $\mathrm{d} \Omega$, et la même bande spectrale $\mathrm{d} \lambda$, à partir de $s(\lambda)$ et du signal de sortie $\mathrm{v}(\lambda)$, il faut tenir compte de l'aire émissive $\mathrm{d} S_{2}$ plus faible que $\mathrm{d} S_{1}$, la diode ayant une hauteur de $5 \mathrm{~mm}$ et ne couvrant pas en totalité le champ utile de hauteur $8 \mathrm{~mm}$.

En supposant l'émission uniforme, la luminance $l(\lambda)$ d'une diode est alors donnée par :

$$
l(\lambda)=\frac{v(\lambda)}{s(\lambda)} \cdot \frac{\mathrm{d} S_{1}}{\mathrm{~d} S_{2}}=1,6 \frac{v(\lambda)}{V(\lambda)} L(\lambda)
$$

et son intensité spectrale $I(\lambda)$ par :

$$
I(\lambda)=\frac{\mathrm{d}^{2} W}{\mathrm{~d} \lambda \mathrm{d} \Omega}=S \cdot l(\lambda)
$$

où $S$ est l'aire totale de la diode : $S=35 \mathrm{~mm}^{2}$.

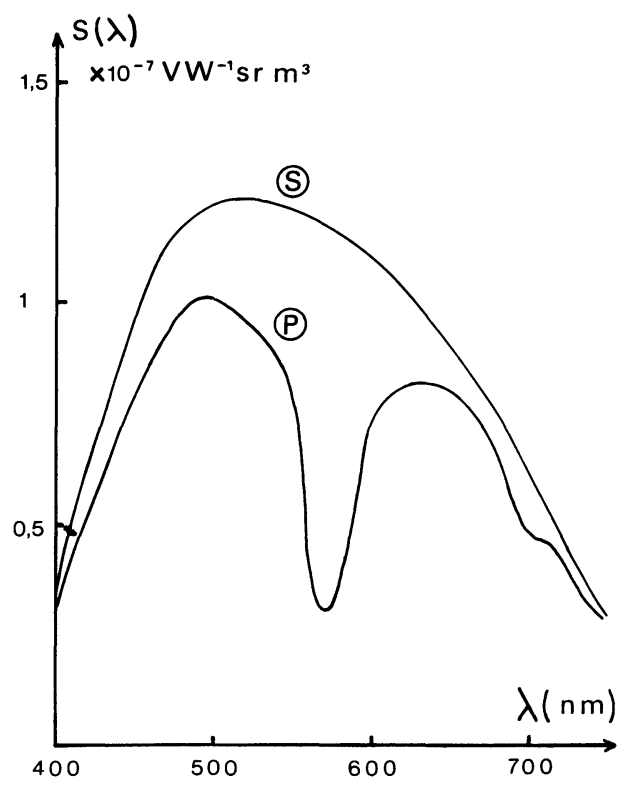

Fig. 2. - Sensibilité du système optique $s(\lambda)$ pour les deux polarisations $\mathrm{s}$ et $\mathrm{p}$ de la lumière incidente.

[Spectral sensitivity $s(\lambda)$ of the optical system for both polarizations $s$ and $\mathrm{p}$ of the incident light.] 
Enfin, une intégration effectuée entre les limites $\lambda_{1}$ et $\lambda_{2}$ du spectre visible donnera l'intensité $I$ émise par la diode :

$$
I=\frac{\mathrm{d} W}{\mathrm{~d} \Omega}\left(\mathrm{W} \mathrm{sr}^{-1}\right)
$$

Notre appareillage ne nous permet d'explorer le diagramme d'émission que dans un plan horizontal, par rotation du support des diodes en face de la fente d'analyse et il n'est donc pas possible de déterminer avec précision le flux total rayonné par la diode.

\section{Caractéristiques du rayonnement émis.}

4. 1 Distributions SPECTRALES. - Pour chaque angle d'analyse $\theta$, défini par la normale $\mathrm{O} y$ à la diode et l'axe du système optique, et pour une valeur fixée de la tension de polarisation $V_{0}$ de la diode, on mesure l'intensité spectrale $I(\lambda)$. La figure 3 donne un exemple de courbes ainsi obtenues pour différentes valeurs de $V_{0}$ avec une diode fabriquée sur un réseau de pas $a=600 \mathrm{~nm}$, et un angle d'observation $\theta=5$ degrés.

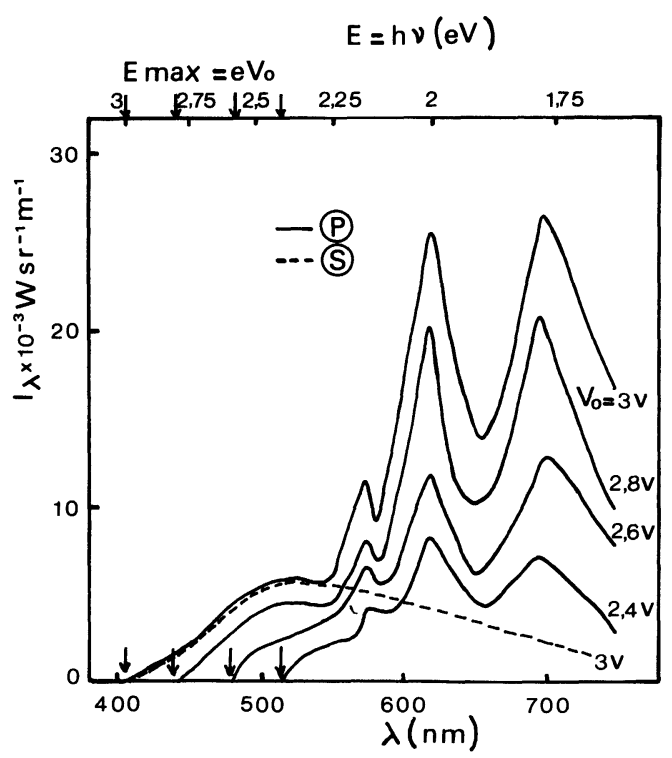

Fig. 3. - Rayonnement émis par une diode MIM sur réseau de pas $a=600 \mathrm{~nm}$ pour plusieurs valeurs de la tension de polarisation. En trait plein : polarisation $p$; en trait discontinu : polarisation s.

[Emission from a MIM diode on grating $(a=600 \mathrm{~nm})$ for various bias voltages.]

En lumière polarisée $s$, on obtient des courbes $I_{s}(\lambda)$ sans singularités, d'amplitude relativement faible. Par contre, en polarisation $p$, les spectres obtenus $I_{p}(\lambda)$ sont d'amplitude nettement plus forte et, en plus d'un fond continu pratiquement équivalent à l'émission polarisée $\mathrm{s}$, on observe plusieurs pics d'importance inégale pour certaines longueurs d'onde caractéristiques.
Ces pics se déplacent en longueur d'onde lorsqu'on fait varier l'angle d'observation $\theta$. Leur amplitude croît proportionnellement au courant $I_{0}$ traversant la diode.

Pour $V_{0}=3 \mathrm{~V}$, le rapport $I_{\mathrm{p}}(\lambda) / I_{\mathrm{S}}(\lambda)$ est égal à 12,5 pour $\lambda=700 \mathrm{~nm}$, avec :

$$
I_{\mathrm{p}}(\lambda) \simeq 20 \times 10^{-3} \mathrm{~W} \mathrm{~m}^{-1} \mathrm{sr}^{-1} .
$$

L'émission de pics de lumière polarisée $\mathrm{p}$ est due aux OPS ayant leur vecteur d'onde $k_{x}$ normal aux traits du réseau. Par contre, le rayonnement observé en polarisation $\mathrm{s}$, et le fond continu polarisé $\mathrm{p}$ ont essentiellement pour origine les rugosités aléatoires qui existent sur la surface du réseau.

Pour une valeur donnée de la longueur d'onde $\lambda$, en polarisation $p$, les courbes donnant $I(\lambda)$ en fonction de l'angle d'observation $\theta$ ont l'allure de celles données sur la figure 4 pour un réseau de pas $a=710 \mathrm{~nm}$, avec $V_{0}=3,25 \mathrm{~V}$. On y observe deux pics importants, obtenus pour des angles symétriques par rapport à $\theta=0$ et correspondant, comme nous le verrons plus loin aux " ordres » $n= \pm 1$ définis par la relation

$$
k_{\|}=k_{0} \sin \theta=k_{x} \pm n k_{\mathrm{R}}
$$

avec $k_{0}=\omega / c=2 \pi / \lambda$ et $k_{\mathrm{R}}=2 \pi / a$.

Le pic $n=+1$ est en général moins intense que le pic $n=-1$.
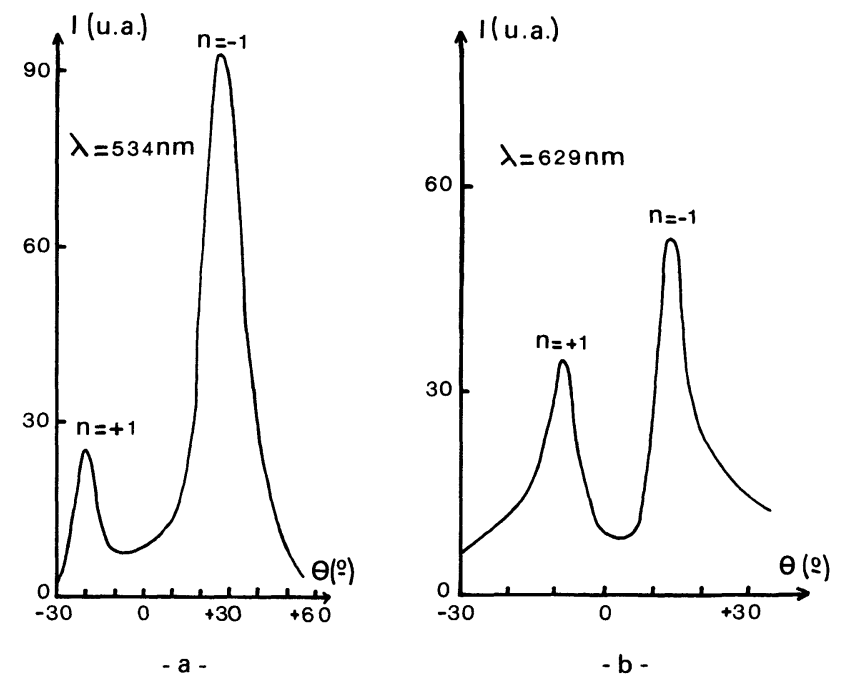

Fig. 4. - Exemple de courbes obtenues par rotation de la diode à longueur d'onde fixe (polarisation $\mathrm{p} ; V_{0}=3,25 \mathrm{~V}$; $a=710 \mathrm{~nm}$ ).

[Example of spectral intensity curves obtained by varying $\theta$ at fixed wavelength (p-polarization; $V_{0}=3.25 \mathrm{~V} ; a=$ $710 \mathrm{~nm})$.]

4.2 Courbe De DISPERSION DES OPS. - A partir des pics obtenus pour une énergie donnée $(\hbar \omega=h c / \lambda)$ et un angle d'observation $\theta$ connu, il est possible d'obtenir la courbe de dispersion des OPS se propa- 
geant sur la surface du réseau dans la direction $\mathrm{Ox}$ normale aux traits de ce réseau.

4.2.1 Réseau de pas $a=540 \mathrm{~nm}$. - La figure 5 représente des spectres obtenus pour $\theta=+20^{\circ}$, $+10^{\circ}$ et $0^{\circ}$. Sur les spectres correspondant à $\theta=20$ et $10^{\circ}$, on peut voir plusieurs pics dont l'intensité augmente lorsque $\theta$ diminue. Pour $\theta=0^{\circ}$, le spectre obtenu ne présente que deux pics, d'amplitude très supérieure à celle des pics observés en $\theta=10^{\circ}$ et $\theta=20^{\circ}$.

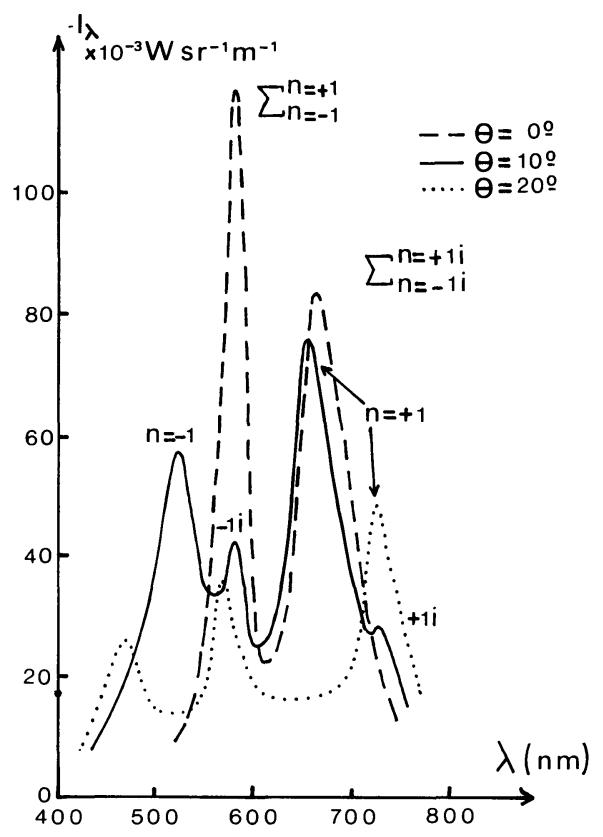

Fig. 5. - Spectres obtenus pour $\theta=0,10$ et 20 degrés. Pour $\theta=0$, on observe l'addition des pics $n= \pm 1$ (OPS externe) et $n= \pm 1 \mathrm{i}$ (mode interne). $V_{0}=3,2 \mathrm{~V} ; I=$ $116 \mathrm{~mA} ; a=540 \mathrm{~nm}$ ).

[Spectra obtained at $\theta=0,10$ and 20 degrees respectively, with $V_{0}=3.2 \mathrm{~V}, I=116 \mathrm{~mA}, a=540 \mathrm{~nm}$. At $\theta=0$, addition of peaks $n= \pm 1$ (external mode) and $n= \pm 1 \mathrm{i}$ (internal mode) can be observed.]

A partir de tels spectres, on peut calculer l'énergie $E(\mathrm{eV})=1240 / \lambda(\mathrm{nm})$ des photons correspondant aux pics, et la composante parallèle à $\mathrm{O} x \mathrm{du}$ vecteur d'onde de l'onde émise :

$$
k_{\|}=\frac{2 \pi}{\lambda} \sin \theta
$$

En portant les valeurs ainsi obtenues dans un diagramme $(E, k)$, on obtient les différentes branches de la courbe de dispersion de la lumière émise, représentées sur la figure 6 . On a également porté sur cette figure les deux branches $\left(k_{x}>0\right.$ et $\left.k_{x}<0\right)$ de la courbe de dispersion des OPS se propageant en sens inverse à l'interface Ag-vide, sur une couche lisse d'Ag de $35 \mathrm{~nm}$ d'épaisseur, et qui sont pratiquement confondues pour $E<1,5 \mathrm{eV}$ avec leur tangente à l'origine. On sait d'autre part que la courbe de disper-

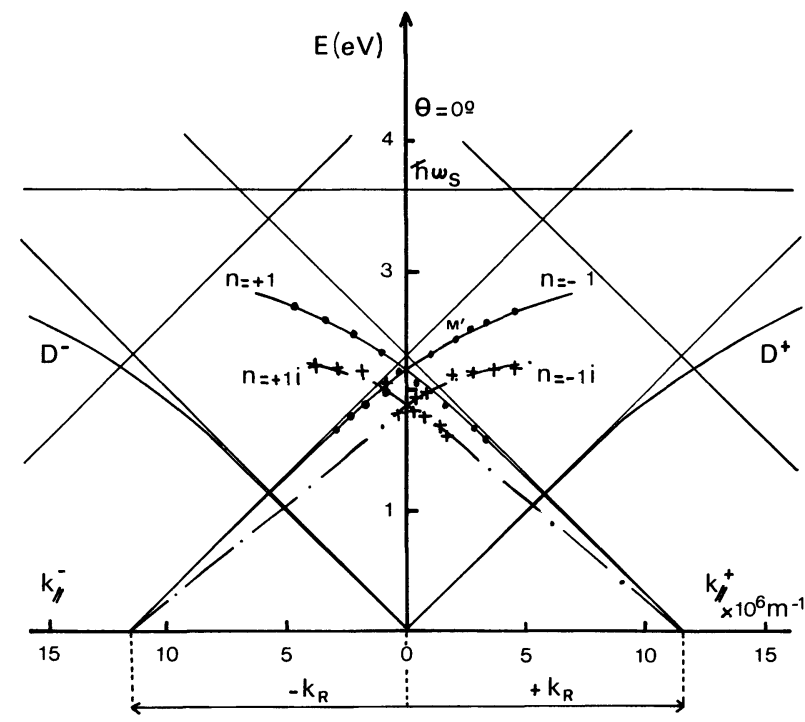

Fig. 6. - Diagramme de dispersion des ondes rayonnées, pour des réseaux de pas $a=540 \mathrm{~nm}\left(k_{\mathrm{R}}=2 \pi / a=11,6 \times\right.$ $\left.10^{6} \mathrm{~m}^{-1}\right)$. L'émission est centrée sur $\theta=0$. Les courbes $n= \pm 1$ correspondent à deux ondes provenant du mode externe, et $n= \pm 1 \mathrm{i}$ au mode interne. $\mathrm{D}^{+}$et $\mathrm{D}^{-}$: courbes de dispersion des OPS externes, déduites des précédentes par translation $\pm k_{\mathrm{R}}$.

[Dispersion diagram of the radiated waves, for grating pitch $a=540 \mathrm{~nm} \quad\left(k_{\mathrm{R}}=2 \pi / a=11.6 \times 10^{6} \mathrm{~m}^{-1}\right)$. Emission takes place around $\theta=0$. Curves $n= \pm 1$ correspond to the external mode, $n= \pm 1 \mathrm{i}$ to the internal mode. $\mathrm{D}^{+}$ and $\mathrm{D}^{-}$are the dispersion curves of surface plasma waves at the Ag-vacuum interface (external mode), obtained by translation $\pm k_{\mathrm{R}}$ of the $n= \pm 1$ curves.]

sion d'une couche faiblement rugueuse est très proche de la précédente, avec un faible décalage $\Delta k_{x}$ vers les grandes valeurs de $k_{x}$, qui est fonction de l'énergie des photons, et reste négligeable pour $E<1,5 \mathrm{eV}$.

Les points expérimentaux s'alignent sur des courbes symétriques par rapport à l'axe $k_{\|}=0$, qui sont pratiquement parallèles aux deux branches des courbes de dispersion de la couche mince d'Ag.

Le décalage entre les courbes de dispersion des OPS et les branches du diagramme de radiation obtenu expérimentalement est égal à un multiple entier du vecteur d'onde $k_{\mathrm{R}}=2 \pi / a$ associé au réseau.

Les pics les plus intenses observés dans les spectres correspondent aux « ordres " $n= \pm 1$ et les branches correspondantes du diagramme de rayonnement se coupent sur l'axe $k_{\|}=0(\theta=0)$ en $E \simeq 2,15 \mathrm{eV}$.

$\mathrm{Si}$ on considère maintenant les pics de faible amplitude de la figure 5, on obtient sur la figure 6 deux autres courbes, situées en dessous des précédentes, qui sont elles aussi symétriques par rapport à $k_{\|}=0$, et dont l'origine est décalée de $\pm k_{\mathrm{R}}$ par rapport à celle des courbes de dispersion des OPS. Les courbes tendent vers une énergie maximum d'environ $2,2 \mathrm{eV}$.

L'origine de ces pics doit être trouvée dans les OPS excitées par les électrons dans la couche d' $\mathrm{Al}_{2} \mathrm{O}_{3}$ 
à l'interface $\mathrm{Ag}-\mathrm{Al}_{2} \mathrm{O}_{3}$. La couche d'Ag étant mince, les champs électromagnétiques associés à cette OPS, qui décroissent de façon exponentielle à partir de l'interface $\mathrm{Al}_{2} \mathrm{O}_{3}-\mathrm{Ag}$, ont encore une amplitude finie à l'interface Ag-vide, et rayonnement directement dans le vide grâce aux reliefs du réseau (Fig. 7).

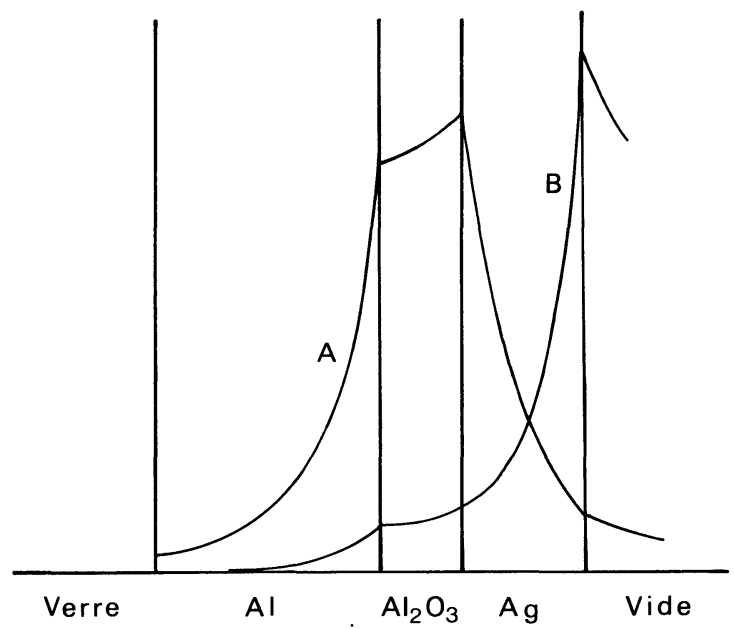

Fig. 7. - Allure de la répartition de l'énergie électromagnétique des OPS. $\mathrm{A}$ : mode interne (interface $\mathrm{Ag}-\mathrm{Al}_{2} \mathrm{O}_{3}$ ); B : mode externe (interface Ag-Vide).

[Schematic representation of the electromagnetic energy density of surface plasma waves excited in the diode. A : internal mode $\left(\mathrm{Ag}-\mathrm{Al}_{2} \mathrm{O}_{3}\right.$ interface). $\mathrm{B}$ : external mode (Agvacuum interface).]

C'est la première fois qu'une contribution non négligeable d'OPS internes est mise en évidence pour des diodes tunnel construites sur réseaux de diffraction. La courbe de dispersion théorique des OPS internes calculée dans [22] met bien en évidence une énergie limite de $2,2 \mathrm{eV}$, mais correspond à des ondes beaucoup plus lentes que celles de la figure 6. L'écart observé ne peut s'expliquer que si l'on affecte à la couche d'alumine une constante diélectrique plus faible que celle utilisée dans les calculs $\left(\varepsilon_{\mathrm{r}}=4\right)$.

On a porté sur la figure 8 l'amplitude des pics associés à l'OPS externe, en fonction de la longueur d'onde à laquelle ils apparaissent lorsqu'on fait varier $\theta$, pour l'ordre $n=-1$ avec $V_{0}=3,2 \mathrm{~V}$ et $I_{0}=116 \mathrm{~mA}$. Le pic maximum très aigu observé en $\lambda \simeq 575 \mathrm{~nm}$ pour $\theta=0$ est dû à l'addition des amplitudes des rayonnements émis par les OPS se propageant en sens inverse sur le réseau, correspondant aux ordres $n= \pm 1$. Les oscillations présentes au voisinage de cette longueur d'onde ont déjà été signalées [28, 29] sans être expliquées. L'émission dans l'ordre $n=-1$ est maximum vers $\lambda \simeq 620 \mathrm{~nm}(h v \simeq 2 \mathrm{eV})$.

4.2.2 Réseau de pas $a=710 \mathrm{~nm}$. - Les spectres ont été relevés entre $\theta_{1}=-29^{\circ}$ et $\theta_{2}=+51^{\circ}$ avec un pas $\theta=2^{\circ}$. En dehors de ce domaine angulaire, les signaux obtenus deviennent très faibles. A partir des différents pics observés pour $V_{0}=3,25 \mathrm{~V}$ et

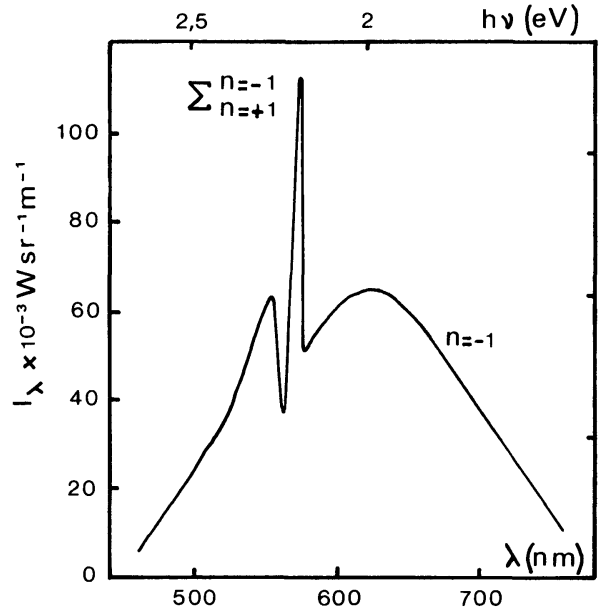

Fig. 8. - Variation de l'amplitude des pics $n=-1$ en fonction de leur longueur d'onde. Le maximum très aigu correspond à l'addition des ordres $n=+1$ et $n=-1$ en $\theta=0 .\left(V_{0}=3,2 \mathrm{~V} ; I=116 \mathrm{~mA} ; a=540 \mathrm{~nm}\right.$.)

[Variation of the amplitude of the peaks $n=-1$ as a function of their wavelength. The sharp maximum corresponds to the addition of the $n= \pm 1$ peaks at $\theta=0$. $\left(V_{0}=3.2 \mathrm{~V} ; I=116 \mathrm{~mA} ; a=540 \mathrm{~nm}\right.$. $\left.)\right]$

$I_{0}=146 \mathrm{~mA}$, on obtient les courbes de dispersion représentées sur la figure 9 en coordonnées $(E, k)$.

En ce qui concerne le rayonnement des OPS externes de l'interface Ag-vide, on constate que, en plus des ordres $n= \pm 1$, les ordres $n= \pm 2$ sont mis en évidence.

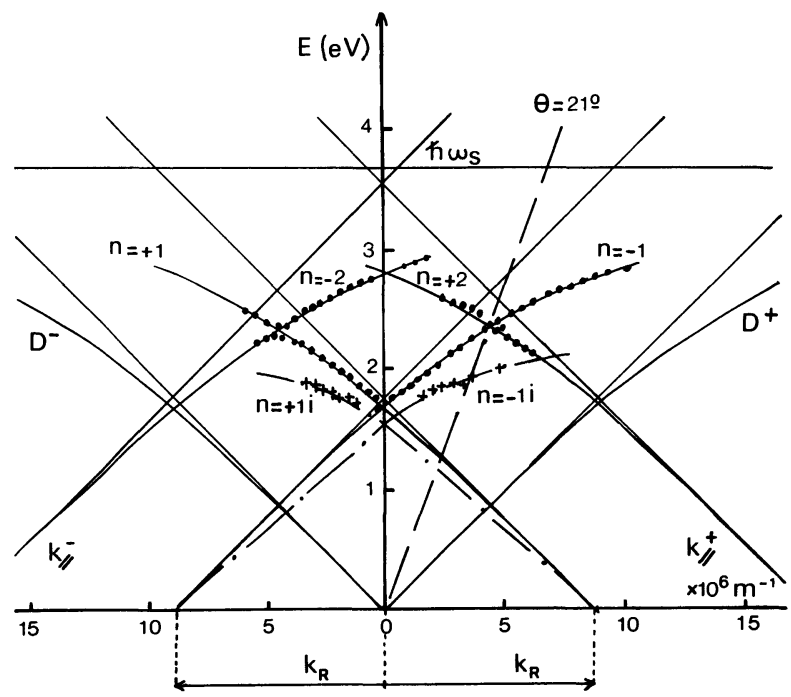

Fig. 9. - Diagramme de dispersion obtenu pour des réseaux de pas $a=710 \mathrm{~nm}\left(k_{\mathrm{R}}=8,85 \times 10^{6} \mathrm{~m}^{-1}\right)$. On observe les ordres $n= \pm 1$ et $n= \pm 2$ du mode externe et les ordres $n= \pm 1 \mathrm{i}$ du mode interne.

[Dispersion diagram obtained for a grating pitch $a=$ $710 \mathrm{~nm}\left(k_{\mathrm{R}}=8.85 \times 10^{6} \mathrm{~m}^{-1}\right)$. Orders $n= \pm 1$ and $n= \pm 2$ of the external mode, $n= \pm 1 \mathrm{i}$ of the internal mode, can be observed.] 
Les branches des courbes de dispersion correspondant aux ordres $n= \pm 1$ et $n= \pm 2$ se croisent respectivement en $\theta=0$ pour $E_{\mathrm{F} 1}=1,75 \mathrm{eV}$ et $E_{ \pm 2}=2,81 \mathrm{eV}$. Les branches correspondant aux ordres $n=-2$ et $n=+1$ d'une part, $n=+2$ et $n=-1$ d'autre part, se croisent respectivement en $\theta=21^{\circ}$ et $E=2,32 \mathrm{eV}$.

La figure 10 montre l'évolution des pics associés aux OPS externes pour les ordres $n=-1$ et $n=+2$, pour 3 valeurs de $\theta=19,21$ et $23^{\circ}$. On observe ici encore l'addition des amplitudes des ondes rayonnées pour $\theta=21^{\circ}$. Un phénomène identique se produit à tous les croisements des différentes branches de la courbe de dispersion.

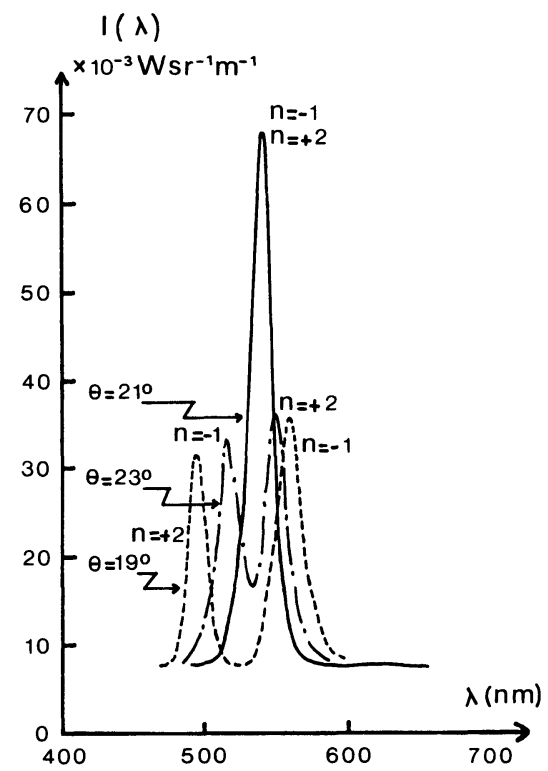

Fig. 10. - Evolution des pics $n=-1$ et $n=+2$ pour $\theta=19,21$ et 23 degrés. Les ordres se croisent en $\theta=21^{\circ}$, avec addition des intensités rayonnées ( $a=710 \mathrm{~nm}$ ).

[Evolution of the peaks $n=-1$ and $n=+2$ for three values of $\theta(\theta=19,21$ and 23 degrees). Modes crossing at $\theta=21$ degrees correspond to an addition of the peaks intensity $(a=710 \mathrm{~nm})$.]

Si l'on excepte la surintensité qui, en principe, est égale à deux fois celle d'un ordre unique, le pic le plus intense correspond à une énergie de $2,18 \mathrm{eV}$ $(\lambda \simeq 570 \mathrm{~nm})$. Au-delà de $E=2,7 \mathrm{eV}$, l'amplitude des pics diminue très rapidement bien que la tension de polarisation $V_{0}$ soit égale à $3,25 \mathrm{~V}$. On peut donc affirmer que le nombre des électrons traversant l'oxyde, puis la couche d'argent, sans subir de perte d'énergie est pratiquement nul.

Pour les OPS internes, les deux branches correspondant aux ordres $n= \pm 1 \mathrm{i}$ ont été de nouveau obtenues. La valeur maximum de l'énergie des photons émis à partir de ces OPS internes serait encore voisine de $2,2 \mathrm{eV}$. Les ordres $n= \pm 2 \mathrm{i}$ 'apparaissent pas, car leur intensité est trop faible pour être détectée sur les enregistrements.

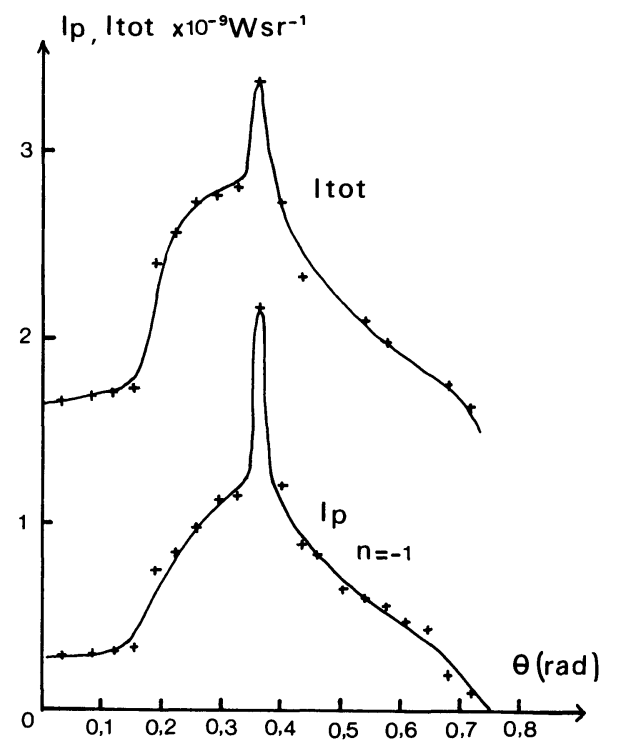

Fig. 11. - Variation de l'intensité associée au pic $n=-1$, et de l'intensité totale rayonnée par une diode MIM en fonction de l'angle d'émission $\theta$. Le maximum très aigu correspond au croisement des ordres $n=-1$ et $n=+2$ en $\theta=21^{\circ}\left(a=710 \mathrm{~nm} ; V_{0}=3,25 \mathrm{~V} ; I=156 \mathrm{~mA}\right)$.

[Variations of the $n=-1$ peak integral intensity $I_{\mathrm{p}}$ and of the total intensity $I_{\text {tot }}$ radiated from a MIM diode as a function of the emission angle $\theta$. The sharp maximum is due to the $n=-1$ and $n=+2$ modes crossing at $\theta=21$ degrees $\left(a=710 \mathrm{~nm} ; V_{0}=3.25 \mathrm{~V} ; I=156 \mathrm{~mA}\right)$.]

\section{Intensité émise et rendement quantique d'émis- sion [32].}

Pour chaque angle $\theta$, et une polarisation $V_{0}$ de l'ordre de 3,2 V, l'analyse du rayonnement fournit un spectre $I_{\lambda}(\lambda)$ s'étendant pratiquement sur tout le domaine visible, et comprenant des pics et un fond continu. Après soustraction du fond continu, on peut déterminer l'aire du pic correspondant à un ordre donné $n=-1$ par exemple, ce qui fournit l'intensité lumineuse $I_{-1}$ rayonnée par l'OPS dans la direction $\theta$.

La figure 11 donne la variation de $I_{-1}$ en fonction de $\theta$ pour le réseau de pas $a=710 \mathrm{~nm}$ avec $V_{0}=3,2 \mathrm{~V}$ et $I_{0}=116 \mathrm{~mA}$. L'intensité est maximale vers $\theta \simeq 20^{\circ}$, et atteint $I_{(-1)_{\max }} \simeq 1,2 \times 10^{-9} \mathrm{~W} \mathrm{sr}^{-1}$. Les réseaux de pas $a=540 \mathrm{~nm}$ conduisent à des courbes semblables ayant leur maximum en $\theta=0^{\circ}$ et telles que $I_{(-1)_{\max }} \simeq 4 \times 10^{-9} \mathrm{~W} \mathrm{sr}^{-1}$.

Par intégration des spectres obtenus, entre $\lambda_{1}=460 \mathrm{~nm}$ et $\lambda_{2}=760 \mathrm{~nm}$, on obtient l'intensité totale rayonnée dans la direction $\theta$. Pour $a=710 \mathrm{~nm}$, cette intensité globale $I_{\text {qot }}$ passe par un maximum en $\theta \simeq 20^{\circ}$, avec $I_{\text {tot }-\max } \simeq 3 \times 10^{-9} \mathrm{~W} \mathrm{sr}^{-1}$. Avec $a=540 \mathrm{~nm}$, on a pour $\theta \simeq 0^{\circ}, I_{\text {tot-max }} \simeq 16 \times$ $10^{-9} \mathrm{~W} \mathrm{sr}^{-1}$.

Bien que la valeur du courant électronique y soit plus faible, l'intensité rayonnée dans l'ordre $n=-1$ par les réseaux de pas $a=540 \mathrm{~nm}$ est 2 à 3 fois plus 
forte que celle obtenue avec les réseaux de pas $a=710 \mathrm{~nm}$. La supériorité des diodes réalisées sur ces réseaux est due essentiellement à la plus grande profondeur $\delta_{0}$ des sillons (on sait en effet [20] que l'intensité rayonnée varie proportionnellement à $\delta_{0}^{2}$, lorsque $\delta_{0}$ est petit devant la longueur d'onde $\lambda$ ).

Le rapport entre les intensités globales maximums rayonnées par les deux types de réseaux est voisin de 5, et pour le réseau de pas $a=540 \mathrm{~nm}$, l'intensité globale maximum est 4 fois plus forte que la contribution du seul ordre $n=-1$ rayonnée par les OPS externes. En admettant que les ordres $n=-1$ et $n=+1$ soient équivalents, on peut en déduire que les ordres supérieurs et le fond continu participent pour moitié au rayonnement global.

Une intégration effectuée sur le pic relatif au seul ordre $n=+1$ du rayonnement des OPS internes, montre que l'intensité maximale $I_{+1}^{\mathrm{i}}$ obtenue au voisinage de $\theta=0$, avec le réseau de pas $a=540 \mathrm{~nm}$, est 2 fois plus faible que celle de l'ordre $n=-1$ des OPS externes.

A partir des valeurs de l'intensité émise dans chaque direction $\theta$, on peut définir deux rendements quantiques de production de photons, exprimés en photons par électron et par stéradian :

- soit un rendement moyen $\varphi_{\mathrm{m}}$, calculé en prenant une valeur moyenne de l'énergie des photons émis dans tout le spectre s'étendant sur le domaine visible $\left(E_{\mathrm{m}} \simeq 2 \mathrm{eV}\right.$ par exemple $)$;

- soit un rendement $\varphi_{\lambda}$ correspondant à un pic de longueur d'onde moyenne $\lambda$ et d'énergie $E=h c / \lambda$.

Pour l'ordre $n=-1$ relatif à l'onde émise par les OPS externes, avec $E \simeq 1,95 \mathrm{eV}$ et $I_{0}=116 \mathrm{~mA}$, on aurait au maximum :

$$
\varphi_{\lambda} \max \simeq 1,7 \times 10^{-8} \text { photons/él-sr }
$$

avec le réseau de pas $a=540 \mathrm{~nm}$.

En prenant $E_{\mathrm{m}}=2 \mathrm{eV}$, on a :

$$
\varphi_{\mathrm{m}} \simeq 6,5 \times 10^{-8} \text { photons/él-sr }
$$

avec ce même réseau.

Ces valeurs sont en bon accord avec celles publiées dans [23] et [28].

Si le rayonnement émis était isotrope dans un angle solide de $2 \pi$ stéradians avec une intensité égale à l'intensité maximum définie précédemment,

$$
I_{\text {tot }-\max } \simeq 16 \times 10^{-9} \mathrm{~W} \mathrm{sr}^{-1}
$$

on aurait, au mieux, une énergie rayonnée de :

$$
W_{\max } \simeq 10^{-7} \text { watts } .
$$

En réalité, seul le fond continu provenant du rayonnement des OPS rendues radiatives par les rugosités aléatoires de surface a un diagramme d'émission pratiquement isotrope. On a vu qu'il contribuait pour environ $50 \%$ à l'énergie émise par la diode. Par contre, le rayonnement émis par les OPS grâce au réseau est très directif. Le diagramme d'émission est symétrique par rapport au plan horizontal $y O x$ normal aux traits du réseau, passant par le centre des diodes. Pour un réseau aux ondulations parfaitement sinusoïdales, il serait symétrique par rapport au plan $z \mathrm{O} y$. On a vu que l'intensité de la radiation émise par les OPS grâce au réseau, était une fonction rapidement variable de $\theta$ et s'annulait pratiquement pour des angles supérieurs à 50 degrés. Dans le cas des réseaux de pas $a=710 \mathrm{~nm}$, l'émission en $\theta=0$ est également négligeable : le diagramme d'émission est alors composé de deux lobes centrés sur $\theta \simeq \pm 23$ degrés. Pour $a=540 \mathrm{~nm}$, on observe au contraire un lobe unique centré $\operatorname{sur} \theta=0$.

Dans tous les cas l'énergie globale rayonnée par une diode est donc inférieure à $10^{-7}$ watts, mais ce facteur est beaucoup moins important, pour une bonne vision de la lumière rayonnée, que l'intensité du rayonnement dans une direction normale à la surface.

\section{Conclusion.}

Nous avons réalisé sur des réseaux holographiques des diodes tunnel $\mathrm{MIM} \mathrm{Al}-\mathrm{Al}_{2} \mathrm{O}_{3}-\mathrm{Ag}$ pouvant supporter sans claquer et durant plusieurs jours de fonctionnement une tension supérieure à $3,2 \mathrm{~V}$, avec une densité de courant importante $\left(j \simeq 0,4\right.$ à $\left.0,5 \mathrm{~A} / \mathrm{cm}^{2}\right)$. L'analyse de leur rayonnement lumineux a bien mis en évidence un fond continu important dû aux rugosités superficielles aléatoires, et les pics rayonnés grâce à une interaction OPS-réseau.

Cette étude a confirmé le rôle des OPS excitées à la surface externe de l'électrode d'argent, et a permis de déceler un rayonnement relativement important provenant des OPS excitées à l'interface interne $\mathrm{Al}_{2} \mathrm{O}_{3}$-Ag.

Nous avons déterminé avec précision les diagrammes de dispersion des différentes ondes émises dans un plan normal aux diodes et aux traits du réseau, et mis en évidence l'addition des intensités rayonnées, aux points de croisement de deux ordres $(n= \pm 1$ et $n= \pm 1$ i en $\theta=0$ pour les réseaux de pas $a=540 \mathrm{~nm}$; $n=-1, n=+2$ et $n=+1, n=-2$ en $\theta= \pm 21^{\circ}$ dans le cas $a=710 \mathrm{~nm}$ ).

Pour nos réseaux qui ont des profondeurs de sillons relativement faibles, $(\delta \leqslant 20 \mathrm{~nm})$, nous n'avons pas observé l'apparition d'une bande interdite étroite aux points de croisement des différents ordres, signalée par d'autres auteurs $[23,27]$ avec des réseaux à modulation plus importante.

Les intensités rayonnées et les rendements quantiques obtenus sont en bon accord avec ceux effectivement mesurés par plusieurs auteurs [23, 28] mais restent encore trop faibles pour rendre possible une application pratique du dispositif.

En effet, la réalisation d'éléments électroluminescents avec des diodes MIM ne peut être envisagée que si l'intensité du rayonnement est accrue par un 
facteur supérieur à $10^{2}$. Cet accroissement pourrait être obtenu en améliorant d'une part le rendement d'excitation des OPS par les électrons, et d'autre part le rendement de conversion OPS-photons rayonnés.

Il faudrait donc pour remplir ces deux conditions : - accroître la tension de fonctionnement et la densité de courant, mais on sera vite limité dans cette voie pàr les contraintes thermiques pouvant entraîner la destruction de la diode;

- supprimer au maximum les chocs inélastiques des électrons dans l'isolant, en améliorant la qualité de cet isolant : un isolant monocristallin serait supérieur à l'isolant amorphe qu'on obtient par oxydation de l'aluminium microcristallin évaporé;

- accroître le libre parcours moyen des électrons dans l'électrode externe, en choisissant une méthode d'évaporation appropriée [24] et en effectuant des recuits;

- diminuer au maximum l'épaisseur de cette électrode externe; mais l'augmentation de résistance va entraîner un échauffement supplémentaire : on pourra l'éviter en renforçant localement l'épaisseur par évaporation de « barres " parallèles;

- accroître la rugosité, jusqu'à l'obtention de la profondeur optimum des sillons, qui correspond à un rendement maximum d'émission d'environ $70 \%$ [34];
- optimiser la forme des sillons, pour accroître le rendement d'émission, car on sait que des réseaux à profil dissymétrique ont des rendements de diffraction supérieurs à ceux des réseaux à profil sinusoïdal [32];

- réduire au maximum les rugosités aléatoires dues vraisemblablement à la résine elle-même [20] afin de diminuer le fond continu et accroître l'intensité dans le lobe utile, par un choix approprié de la résine et du mode de développement ;

- concentrer au maximum la lumière dans un lobe centré sur la normale à la surface, par un choix judicieux du pas du réseau. On a vu qu'il valait mieux utiliser des réseaux de pas voisin de $0,5 \mu \mathrm{m}$ pour amener le maximum d'émission en $\theta=0$;

- enfin, les diodes devront être réalisées directement sur support métallique bien refroidi, afin de pouvoir obtenir un fonctionnement à la température ambiante.

\section{Remerciements.}

Les auteurs adressent leurs plus vifs remerciements au Professeur J. Marcou du Laboratoire d'Opto-AcoustoElectronique de l'Université de Valenciennes, qui leur a remis gracieusement de nombreux réseaux holographiques réalisés par ses soins.

\section{Bibliographie}

[1] Нicкмотт, T. W., J. Appl. Phys. 33 (1962) 2669, 35 (1964) 2118 et 2679, 36 (1965) 1885; Thin Solid Films 9 (1972) 431.

[2] Kanter, M., Feibelman, W. A., J. Appl. Phys. 34 (1963) 1569.

[3] Pong, W., Inouye, C., Matsunaga, F., Moriwaki, M., J. Appl. Phys. 46 (1975) 2310.

[4] Delaunay, G., Despujols, J., Thin Solid Films 51 (1978) 227

[5] Delaunay, G., Despujols, J., Thin Solid Films 76 (1981) 49.

[6] Lambe, J., Mc Carthy, S. J., Phys. Rev. Lett. 37 (1976) 923.

[7] Mc Carthy, S. J., Lambe, J., Appl. Phys. Lett. 30 (1977) 427.

[8] Economou, E. N., Phys. Rev. 182 (1969) 539.

[9] Davis, L. C., Phys. Rev. B 16 (1977) 2482.

[10] LaKs, B., Mills, D. L., Phys. Rev. B 20 (1979) 4962.

[11] Laks, B., Mills, D. L., Phys. Rev. B 21 (1980) 5175.

[12] Jain, R. K., Wagner, S., Olson, D. H., Appl. Phys. Lett. 32 (1978) 62.

[13] Hansma, P. K., Broida, H. P., Appl. Phys. Lett. 32 (1978) 545.

[14] Hone, D., Muhlschlegel, B., Scalapino, D. J., Appl. Phys. Lett. 32 (1978) 203.

[15] Rendell, R. W., Scalapino, D. J., Muhlschlegel, B., Phys. Rev. Lett. 41 (1978) 1746.

[16] Adams, A., Wyss, J. C., Hansma, P. K., Phys. Rev. Lett. 42 (1979) 912.

[17] Adams, A., Hansma, P. K., Phys. Rev. B 23 (1981) 3597.
[18] Parvin, K., PARker, W., Solid State Commun. 37 (1980) 629.

[19] Kroó, N., Szentirmay, Zs, Felszerfalvi, J., Phys. Status Solidi B 102 (1980) 227.

[20] Kirtley, J. R., Theis, T. N., Tsang, J. C., Appl. Phys. Lett. 37 (1980) 435.

[21] Kroó, H., Szentirmay, Zs, Felszerfalvi, J., Phys. Lett. A 81 (1981) 399.

[22] Laks, B., Mills, D. L., Phys. Rev. B 22 (1980) 5723.

[23] Kirtley, J. R., Theis, T. N., Tsang, J. C., Phys. Rev. B 24 (1981) 5650

[24] Kirtley, J. R., Theis, T. N., Tsang, J. C., Di Maria, D. T., Phys. Rev. B 27 (1983) 4601.

[25] Walmsley, D. G., Quinn, H. F., Dawson, P., Phys. Rev. Lett. 49 (1982) 892.

[26] Dawson, P., Ferguson, A. J. L., Walmsley, D. G., Solid State Commun. 44 (1982) 1127.

[27] Kroó, N., Szentirmay, Zs, Felszerfalvi, J., Phys. Lett. A 86 (1981) 445.

[28] Kroó, N., Szentirmay, Zs, Felszerfalvi, J., Phys. Lett. A 88 (1982) 90.

[29] Moulessehoul, S. M., Thèse Doctorat 3e cycle, Orsay 1983.

[30] Moulessehoul, S. M., Septier, A., Marcou, J., C. R. Hebd. Séan. Acad. Sci. Sér. II (1983) 441.

[31] Heitmann, D., Opt. Commun. 20 (1977) 292.

[32] Moulessehoul, S. M., Septier, A., V Int. Conf. Sol. Surf., Madrid, Paper no TF4-OR8 (1983).

[33] Mashev, L., Tonchev, S., Opt. Commun. 47 (1983) 5.

[34] Moreland, J., Adams, A., Hansma, P. K., Phys. Rev. 25 (1982) 2297. 